\title{
MALARIA SITUATION IN BULGARIA AND SURVEILLANCE MEASURES (1991-2000)
}

\section{R.I. KURDOVA* \\ D.I. VUTCHEV
P.P PETROV \\ D.I. VUTCHEV
P.P PETROV}

Received: 20/03/01

Accepted: 03/06/02
National Center of Infectious and Parasitic Diseases

26, YankoSakazov Blvd., 1504, Sofia, Bulgaria

* to whom all correspondence should be addressed e-mail: kurdova@ncipd.netbg.com

\begin{abstract}
The malaria situation in Bulgaria during the past decade of the century was analysed and the evaluation of the risk of re-emerging of the diseases and factors determining it in the phase of maintanence of malaria eradication was carried out. The epidemiological analysis of malaria cases notified during the period 1991-2000 in Bulgaria is presented. Of a total of 382 malaria episodes registered, 363 (95.03\%), were imported into Bulgaria from endemic countries, $1(0.26 \%)$ occurred after haemotransfusion and $18(4.71 \%$ ) were indigenous introduced ones. Plasmodium falciparum accounted for $68.59 \%$ of all imported cases, P.vivax, for $23.82 \%$, P.ovale, for $2.36 \%$, P.malariae, for $1.85 \%$. In $1.83 \%$ more than one species was detected, and in 6 cases, the species was not identified. The import of malaria came mainly from Africa-299 (82.37\%) cases, followed by Asia-63 (17.36\%) and Oceania-1 (0.27\%). Four cases with falciparum malaria resulted in death. A great number of imported cases-231 (63.81\%) was diagnosed during the potential malaria season in Bulgaria (April-October). The analysis of present entomological situation showed a high density of Anopheles population. As an indication for existence of potential risk of spread of indigenous malaria, especially in cases of delayed diagnosis and treatment, 18 indigenous cases of vivax malaria were recorded in 1995-1996 in the region of the town of Sandanski, for the first time after malaria eradication in 1965. The cases were classified as introduced ones. The analysis of the essential malaria import in Bulgaria, climatic conditions and presence of Anopheline species revealed a fairly high level of malariogenic potential. The stratification of the country was carried out in accordance with the degree of hazard of malaria spreading, and the territory was divided into three categories of regions: with high-, medium- and low-risk. The present situation, that probably will be valid for the future, too, requires a scientific based prognosis and improvement of a system of surveillance activities aiming at prevention of indigenous endemic and epidemic spreading of malaria in the country, as well as elaboration of adequate activities of the health network in case of reappearance of autochthonous cases.
\end{abstract}

KEY WORDS: Imported malaria, introduced malaria, induced malaria, entomological situation, malaria surveillance, Bulgaria 


\section{INTRODUCTION}

The Balkan Peninsula has been endemic for malaria since ancient times. Malaria was widely distributed in Bulgaria, too. During the period of 1922-1930, the annual number of malaria cases registered increased from 1,100 to 70,000 (Stanchev and Todorov, 1967; Petrov, 1979; 1990; 1997). The highest morbidity rate (2066\%ooo) with 144,632 malaria patients diagnosed was established in 1946. The renewed malaria eradication efforts (including mosquito vector control, case detection and treatment, and chemoprophylaxis) initiated in 1948, led to malaria eradication as an autochthonous disease in Bulgaria in 1965. Since that time, until 1995, mainly cases imported from malaria endemic countries have been recorded (Petrov, 1979; 1990; 1997; Petrov and Kurdova-Mintcheva, 1984; 1987). Induced malaria cases and relapses were sporadically found, all of them caused by P.malariae.

During the period of 1966-2000, a total number of 2,850 imported malaria cases resulting from infection with P.vivax, P.falciparum, P.malariae and P.ovale were registered, with nine deaths (Petrov, 1979; 1990; 1997; Vutchev and Kurdova, 1999, Kurdova et al., 2000; Petrov et al., 2000).

During the past decade of the century, on the background of the democratization of the society and socio-economical changes in the country, new trends in the epidemiological characteristics of malaria were revealed, although it remained the major disease among other imported infectious and parasitic diseases.

The objective of the study was to analyse the malaria situation in Bulgaria during the past decade of the century and to evaluate the risk of re-emerging of the diseases and factors determining it in the the phase of maintanence of malaria eradication.

The official data of malaria cases notified during the period 1991-2000 in Bulgaria were analyzed. The data were collected by the Departments of Medical Parasitology at District Hygiene Epidemiological Inspections in the country and sent to the Ministry of Health (MH) and to the National Centre of Infectious and Parasitic Diseases (NCIPD) in Sofia. In all cases, diagnosis and species identification were confirmed by the Department of Parasitology and Tropical Medicine at NCIPD. Demographic, epidemiological and clinical information on malaria cases was recorded on a special data collection form. Data on climatic conditions and Anopheline situation in the country regions were also collected and analyzed.

\section{RESULTS AND DISCUSSION}

\section{Malaria situation during the past 10 years (1991-2000).}

Of a total of 382 malaria episodes registered in the period 1991-2000, the greatest number - 363 $(95.03 \%)$, were imported into Bulgaria from endemic countries, $1(0,26 \%)$ occurred after haemotransfusion and $18(4.71 \%)$ were indigenous introduced ones.

\section{Imported malaria}

The malaria import in the mentioned decade (363 cases) is greater than during the decade following the eradication of the disease (1966-1975, 132 cases) (Petrov, 1979; 1990; Stanchev and Todorov, 1967). At the same time, there is presently a reduction in the number of cases in comparison with the consecutive periods: 19761985 (1,154 cases), 1986-1990 (471 cases for the 5 years period). This reduction could be explained by the decreased number of foreign workers entering Bulgaria from malaria-endemic countries during these past years (Petrov, 1979; 1990; 1997; Petrov and Kurdova-Mintcheva, 1984; 1987).

The annual number of imported malaria cases varies with peaks registered in 1991 (49 cases), 1994 (62) and 1999 (47), and an average of 36.3 cases per year (Fig. 1). Plasmodium falciparum accounted for $68.59 \%$ of all imported cases, P.vivax for $23.82 \%$, P.ovale for $2.36 \%$ and P.malariae for $1.85 \%$ (Table 1). In $1.83 \%$ of the cases, more than one species was detected, and in 6 cases, the species was not identified because of the urgent treatment carried out, based on clinical indications. A typical feature of the previous periods was the predominating import of vivax malaria (Petrov, 1979; 1990; 1997; Petrov and Kurdova-Mintcheva, 1984; 1987; Stanchev and Todorov, 1967).

There is also a new trend in the continents where the imported cases were acquired. While in the past the import of malaria came mainly from Asia, nowadays it is mostly from Africa-299 $(82.37 \%)$ cases, followed by Asia-63 (17.36\%) 


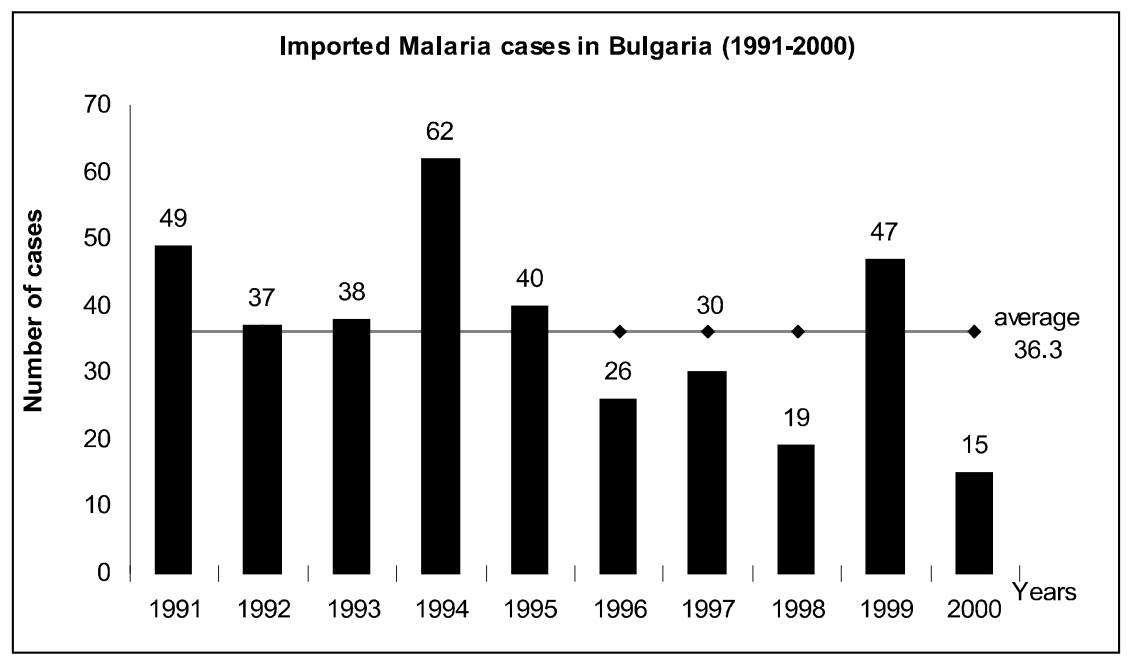

Figure 1. Imported malaria cases in Bulgaria (1991-2000)

and Oceania-1 (0.27\%) (Petrov, 1979; 1990; 1997; Petrov and Kurdova-Mintcheva, 1984; 1987), (Table 2). Malaria was imported from 43 countries altogether-27 African countries, 15 Asian and Oceania. Leading this list were Nigeria, Angola, Cameroon, Sudan, India etc. (Table 2). Most of the patients (91.60\%) with falciparum malaria acquired the infection in Africa, mainly in hyperendemic countries like Nigeria, Angola, Cameroon, Sudan, etc., the rest $(8.02 \%)$ were infected in Asia and one $(0.38 \%)$ got infected in Oceania (Table 2). During the past 10 years, $53.42 \%$ of the cases with vivax malaria originated in Asia (India, Cambodia, Pakistan, Afghanistan, etc.) and $46.58 \%$ - in Africa (Sudan, Nigeria, Angola, etc.). P.ovale infections were imported solely from Africa, whereas P.malariae infectionsfrom both Africa and Asia.

During the previous years mainly foreigners imported malaria into Bulgaria (Petrov, 1979; 1990; 1997; Petrov and Kurdova-Mintcheva, 1984; 1987). In the past decade, the ratio between foreigners and locals importing the disease changed. Of 363 cases, $70.25 \%$ were imported by Bulgarian citizens (Table 3$)$. The majority $(54.12 \%)$ consisted of members of sea or air crews and drivers of

Table 1. Malaria cases classified by species of plasmodia and number of deaths in Bulgaria (1991-2000)

\begin{tabular}{|c|c|c|c|c|c|c|c|c|}
\hline \multirow{3}{*}{$\begin{array}{l}\text { Species of } \\
\text { Plasmodia }\end{array}$} & \multirow{2}{*}{\multicolumn{2}{|c|}{ Number of cases }} & \multicolumn{5}{|c|}{ Classification } & \multirow{3}{*}{$\begin{array}{c}\text { Number o } \\
\text { deaths }\end{array}$} \\
\hline & & & \multicolumn{2}{|c|}{ Imported } & \multicolumn{2}{|c|}{ Autochthonous } & \multirow[t]{2}{*}{ Induced } & \\
\hline & No & $\%$ & No & $\%$ & No & $\%$ & & \\
\hline P.vivax & 91 & 23.82 & 73 & 20.11 & 18 & 100 & & \\
\hline P.falciparum & 262 & 68.59 & 262 & 72.17 & & & & 4 \\
\hline P.malariae & 7 & 1.83 & 6 & 1.65 & & & 1 & \\
\hline P.ovale & 9 & 2.36 & 9 & 2.48 & & & & \\
\hline $\begin{array}{l}\text { P.falciparum } \\
+ \text { P.vivax }\end{array}$ & 5 & 1.31 & 5 & 1.38 & & & & \\
\hline $\begin{array}{l}\text { P.falciparum } \\
+ \text { P.ovale }\end{array}$ & 1 & 0.26 & 1 & 0.27 & & & & \\
\hline $\begin{array}{l}\text { P.vivax }+ \\
\text { P.malariae }\end{array}$ & 1 & 0.26 & 1 & 0.27 & & & & \\
\hline Unknown & 6 & 1.57 & 6 & 1.65 & & & & \\
\hline Total & 382 & 100.00 & 363 & 100.00 & 18 & 100 & 1 & 4 \\
\hline
\end{tabular}


Table 2. Imported malaria cases by continents and countries, where infection was acquired (1991-2000)

\begin{tabular}{|c|c|c|c|c|c|c|c|c|c|c|}
\hline \multirow[b]{2}{*}{ No. } & \multirow[b]{2}{*}{ Origine } & \multirow[b]{2}{*}{$\begin{array}{l}\text { Number } \\
\text { of Cases }\end{array}$} & \multicolumn{8}{|c|}{ Species of Plasmodia } \\
\hline & & & P.vivax & P.falciparum & P.malariae & P.ovale & $\begin{array}{c}\text { P.falciparum } \\
+ \text { P.vivax }\end{array}$ & $\begin{array}{c}\text { P.falciparum } \\
+ \text { P.ovale }\end{array}$ & $\begin{array}{l}\text { P.vivax }+ \\
\text { P.malariae }\end{array}$ & Unknown \\
\hline & Africa & & & & & & & & & \\
\hline 1 & Nigeria & 95 & 7 & 80 & 2 & 3 & 1 & 1 & 1 & \\
\hline 2 & Angola & 44 & 6 & 34 & & 3 & & & & 1 \\
\hline 3 & Cameroon & 36 & 1 & 30 & & & & & & 5 \\
\hline 4 & \begin{tabular}{|l|} 
Sudan \\
\end{tabular} & 29 & 14 & 15 & & & & & & \\
\hline 5 & Benin & 14 & & 14 & & & & & & \\
\hline 6 & Ghana & 10 & 1 & 8 & & 1 & & & & \\
\hline 7 & Guinea & 9 & & 8 & & 1 & & & & \\
\hline 8 & Zaire & 9 & & 8 & & & 1 & & & \\
\hline 9 & \begin{tabular}{|l|} 
Côte \\
d'Ivoire
\end{tabular} & 7 & 7 & & & & & & & \\
\hline 10 & \begin{tabular}{|l|} 
Congo \\
\end{tabular} & 5 & 2 & 2 & & & 1 & & & \\
\hline 11 & Mozambique & 5 & & 4 & 1 & & & & & \\
\hline 12 & Zimbabwe & 5 & & 5 & & & & & & \\
\hline 13 & Gabon & 4 & & 4 & & & & & & \\
\hline 14 & Senegal & 3 & & 3 & & & & & & \\
\hline 15 & Gambia & 3 & & 3 & & & & & & \\
\hline 16 & Mauritania & 3 & & 1 & & 1 & 1 & & & \\
\hline 17 & Tanzania & 3 & & 2 & 1 & & & & & \\
\hline 18 & $\begin{array}{l}\text { Equatorial } \\
\text { Guinea }\end{array}$ & 2 & & 2 & & & & & & \\
\hline 19 & Ethiopia & 2 & 1 & 1 & & & & & & \\
\hline 20 & Egypt & 2 & & 2 & & & & & & \\
\hline 21 & Uganda & 2 & & 2 & & & & & & \\
\hline 22 & Kenia & 2 & & 2 & & & & & & \\
\hline 23 & Liberia & 1 & & 1 & & & & & & \\
\hline 24 & Somalia & 1 & 1 & & & & & & & \\
\hline 25 & Mali & 1 & 1 & & & & & & & \\
\hline 26 & Rwanda & 1 & & 1 & & & & & & \\
\hline \multirow[t]{3}{*}{27} & Togo & 1 & & 1 & & & & & & \\
\hline & Sub-Total & 299 & 34 & 240 & 4 & 9 & 4 & 1 & 1 & 6 \\
\hline & Asia & & & & & & & & & \\
\hline 28 & India & 17 & 12 & 5 & & & & & & \\
\hline 29 & Cambodia & 10 & 4 & 6 & & & & & & \\
\hline 30 & Iran & 5 & 2 & 3 & & & & & & \\
\hline 31 & Pakistan & 5 & 4 & & 1 & & & & & \\
\hline 32 & Afghanistan & 5 & 3 & 1 & 1 & & & & & \\
\hline 33 & Syria & 3 & 3 & & & & & & & \\
\hline 34 & Iraq & 3 & 2 & 1 & & & & & & \\
\hline 35 & Turkey & 3 & 3 & & & & & & & \\
\hline 36 & Sri Lanka & 3 & 1 & 2 & & & & & & \\
\hline 37 & Bangladesh & 2 & 1 & 1 & & & & & & \\
\hline 38 & Vietnam & 2 & 1 & & & & 1 & & & \\
\hline 39 & Indonesia & 2 & 1 & 1 & & & & & & \\
\hline 40 & Yemen & 1 & 1 & & & & & & & \\
\hline 41 & $\begin{array}{l}\text { Lao People's } \\
\text { DR }\end{array}$ & 1 & 1 & & & & & & & \\
\hline \multirow[t]{3}{*}{42} & Saudi Arabia & 1 & & 1 & & & & & & \\
\hline & Sub-Total & 63 & 39 & 21 & 2 & & 1 & & & \\
\hline & Oceania & & & & & & & & & \\
\hline \multirow[t]{3}{*}{43} & $\begin{array}{l}\text { Papua-New } \\
\text { Guinea }\end{array}$ & 1 & 1 & & & & & & & \\
\hline & Sub-Total & 1 & & 1 & & & & & & \\
\hline & Total & 363 & 73 & 262 & 6 & 9 & 5 & 1 & 1 & 6 \\
\hline
\end{tabular}


Table 3. Imported malaria cases by population groups (1991-2000)

\begin{tabular}{|l|c|c|c|c|c|c|}
\hline \multirow{2}{*}{ Population Group } & \multicolumn{2}{|c|}{ Foreigners } & Bulgarian nationals & \multicolumn{2}{c|}{ Total } \\
\cline { 2 - 7 } & No. & $\mathbf{\%}$ & No. & \% & No. & \% \\
\hline Immigrants \& Refugees & 5 & 4.63 & & & 5 & 1.38 \\
\hline Professionals \& laborers & 14 & 12.96 & 77 & 30.20 & 91 & 25.06 \\
\hline Students & 52 & 48.15 & 2 & 0.78 & 54 & 14.88 \\
\hline Military personnel & 1 & 0.93 & 10 & 3.92 & 11 & 3.03 \\
\hline Tourists & 27 & 25.00 & 28 & 10.98 & 55 & 15.15 \\
\hline $\begin{array}{l}\text { Sea or air crews, drivers of } \\
\text { international transport vehicles }\end{array}$ & 9 & 8.33 & 138 & 54.12 & 147 & 40.50 \\
\hline Total & $\mathbf{1 0 8}$ & $\mathbf{2 9 . 7 5}$ & $\mathbf{2 5 5}$ & $\mathbf{7 0 . 2 5}$ & $\mathbf{3 6 3}$ & $\mathbf{1 0 0}$ \\
\hline
\end{tabular}

Table 4. Registered malaria cases by months (1991-2000)

\begin{tabular}{|c|c|c|c|c|c|c|c|c|c|c|c|c|}
\hline Months & Jan. & Feb. & Mar. & Apr. & May & June & July & Aug. & Sept. & Oct. & Nov. & Dec. \\
\hline $\begin{array}{c}\text { Number } \\
\text { of cases }\end{array}$ & 28 & 29 & 10 & 29 & 34 & 41 & 45 & 31 & 25 & 34 & 24 & 33 \\
\hline & \multicolumn{10}{|c|}{ कotential malaria season } \\
\hline
\end{tabular}

international transport vehicles. The rest included other professionals and laborers working in endemic countries $(30.20 \%)$, tourists $(10.98 \%)$, military personnel $(3.92 \%)$ and students $(0.78 \%)$. Among the $108(29.75 \%)$ foreigners, the largest group of patients $(48.15 \%)$ consisted of students, followed by tourists $(25.00 \%)$, professionals and laborers $(12.96 \%)$, immigrants and refugees $(4.63 \%)$ and military personnel $(0.93 \%)$.

Infected males accounted for $85.67 \%$ of all imported cases, and females - for $14.33 \%$ respectively. Most affected were people over 21 years old (Fig. 2).

A great number of imported cases-231 (63.81\%) were diagnosed during the potential malaria season in Bulgaria (April-October). This is an indication of the existence of potential risk for spread of indigenous malaria, especially in cases of delayed diagnosis and treatment (Table 4). In this respect, there is an essential hazard in the former malaria regions on the Black Sea coast (Burgas, Varna), in the Danube plain (Russe) and the Tracian plain (Plovdiv), where 152 (41.99\%) cases were registered during the period of April-October.

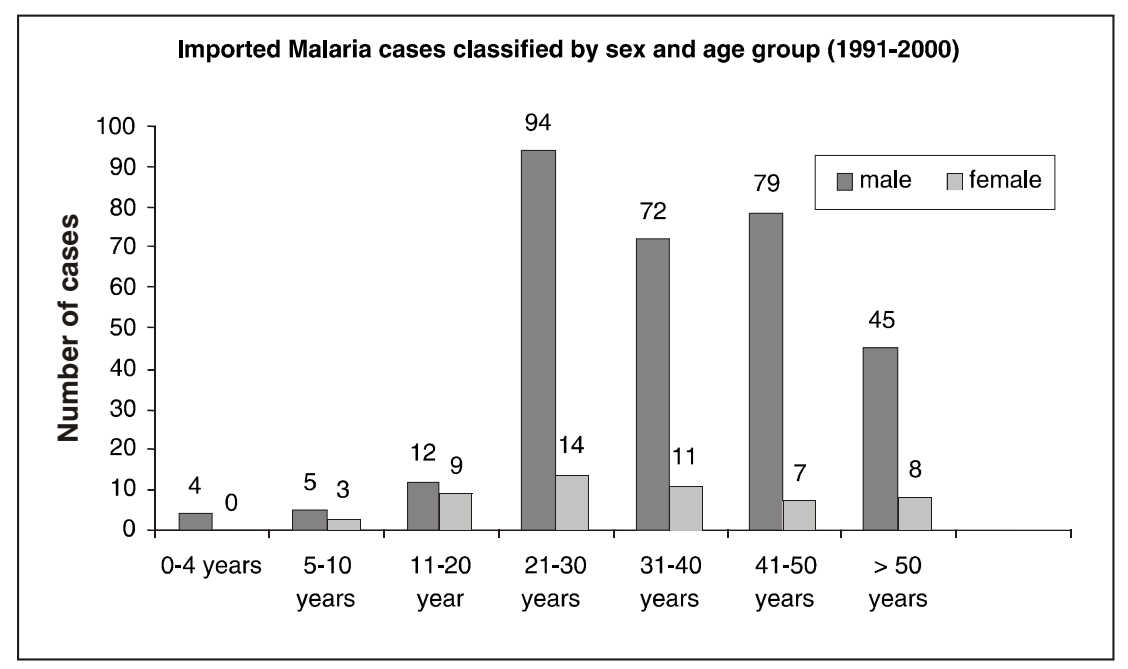

Figure 2. Imported malaria cases classified by sex and age group (1991-2000) 
As a result of delayed diagnosis and appropriate etiological treatment due to belated seeking of medical assistance, 4 cases (2 Bulgarians and 2 foreigners) with Malaria tropica resulted in death. (Table 1).

\section{Induced malaria}

Only one case of Malaria quartana after haemotransfusion was reported during the period (Table 1).

\section{Indigenous malaria}

In 1995, in four settlements in the region of the town of Sandanski situated in South-Western Bulgaria, for the first time after malaria eradication in 1965, 11 indigenous cases of vivax malaria were registered. The cases were classified as introduced ones. The patients diagnosed had never traveled abroad. The epidemiological investigation carried out revealed that the source of infection of the local mosquito populations (A.maculipennis) were immigrants from Africa who stayed in the Struma river valley for several weeks before illegally crossing the border with Greece. In the next year (1996), 7 additional vivax malaria cases were diagnosed in inhabitants of the same settlements, probably due to infection with a strain characterized by long incubation period. All the patients were duly diagnosed, hospitalized and radically treated with Chloroquine and Primaquine.

The eradication of the malaria outbreak was organized and carried out by parasitologists from the National Center of Infectious and Parasitic Diseases in Sofia with the assistance of the local health network. In accordance with the control program elaborated for this Southern region of the country and especially for the settlements mentioned, this area is under permanent surveillance. As a result of these activities, no other cases were detected in the following years.

\section{Present entomological situation}

Of the 40 mosquito species identified in the country, 8 belonged to the genus Anopheles (Christova, 1980). During the pre-eradication period, the predominating mosquito species involved in malaria transmission were A.maculipennis, A.sacharovi and A.superpictus. The same holds for the present. The seasonal activity of the mosquitoes is manifested during May-
October with a maximum in August for most parts of the country. Recently, Anopheles distribution has considerably changed. Thousands of acres of swamps were drained, but numerous artificial ponds were built and large stretches of cultivated land are irrigated. There are other breeding places of anopheline mosquitoes. For these reasons the density of Anopheles populations has not decreased but actually even increased in some districts.

Studies on adult mosquito (imago) susceptibility and of mosquito larvae to insecticides have been going on since 1960 using standard methods recommended by WHO (Petrov and KurdovaMintcheva, 1984; 1987; Christova, 1980). Resistance to dieldrin was established. During the past decades individual populations in some country districts exhibit tolerance or resistance to the following preparations: tolerance to hexachloran, marginal resistance to bromophos and from tolerance to resistance to fenitrothion. Susceptibility to the organophosphorus carbamite and pyrethroids is within normal limits.

A well organized vector control network on national (National Centre of infectious and Parasitic Diseases, Sofia) and regional level (regional vector control stations) was set up under the management of the Ministry of Health, whose principal task is to control all kinds of insect vectors of human diseases. Mosquito control is not carried out on a large scale but is mainly limited to resort regions in order to ensure peoples' well-being at work and leisure. Biological methods of control such as breeding of Gambusia affinis and carps are also widely applied.

\section{Surveillance measures}

The surveillance of malaria is mainly an operative action presented by a system of complex measures including contingents at risk registration, diagnosis, treatment, prophylaxis and antiepidemic activity regarding imported and indigenous malaria cases. The malaria import in the country is under surveillance so the dynamics of the process is under constant follow up, aiming at readiness for preventive actions.

With this aim in mind and in accordance with the degree of hazard of malaria spreading, the country was divided into three categories of regions: with high-, medium- and low-risk. The division is based on three criteria: 
Table 5. Risk of vivax malaria spreading in various regions of Bulgaria

\begin{tabular}{|c|c|c|c|c|}
\hline Risk factor & $\begin{array}{c}\text { Period of effec- } \\
\text { tive infection } \\
\text { of mosquitoes } \\
\text { (days) }\end{array}$ & $\begin{array}{c}\text { Potential } \\
\text { turnovers }\end{array}$ & Vectors & Regions \\
\hline High & $120-160$ & $4-5$ & $\begin{array}{c}\text { A.maculipennis } \\
\text { A.superpictus }\end{array}$ & $\begin{array}{c}\text { Blagoevgrad, Burgas, Varna, V.Turnovo, Vidin, } \\
\text { Kardjali, Pazardjik, Pleven, Plovdiv, Russe, } \\
\text { Sliven, Stara Zagora, Haskovo, Jambol, } \\
\text { Montana, Vratza } \\
\text { (16) }\end{array}$ \\
\hline Medium & $100-120$ & $3-4$ & A.maculipennis & $\begin{array}{c}\text { Gabrovo, Kjustendil, Lovethc, Razgrad, Silistra, } \\
\text { Sofia, Dobtitch, Targovishte, Shumen } \\
\text { (9) }\end{array}$ \\
\hline Low & $50-100$ & $1-3$ & A.maculipennis & Pernik, Smolian, Sofia - city (3) \\
\hline
\end{tabular}

1. The duration (in days) of the period of effective infection of mosquitoes, e.g. the period of time when there is a favorable temperature range enabling sporogony to be completed in the mosquito. The potential malaria season in Bulgaria is about 7 months covering the period of April-October.

2. The number of turnovers of malaria infection, e.g. the period of time including sporogony and epidemiological incubation (appearance of sexual stages of plasmodia in human peripheral blood).

3. The vector species. The territorial division is presented in Table 5 and Fig. 3.

Based on the evaluation of the real hazard of malaria reintroduction, the Ministry of Health carries out a malaria surveillance system aiming at checking malaria import, and monitoring and prevention of indigenous distribution. The system was established and has been in effect from 1978

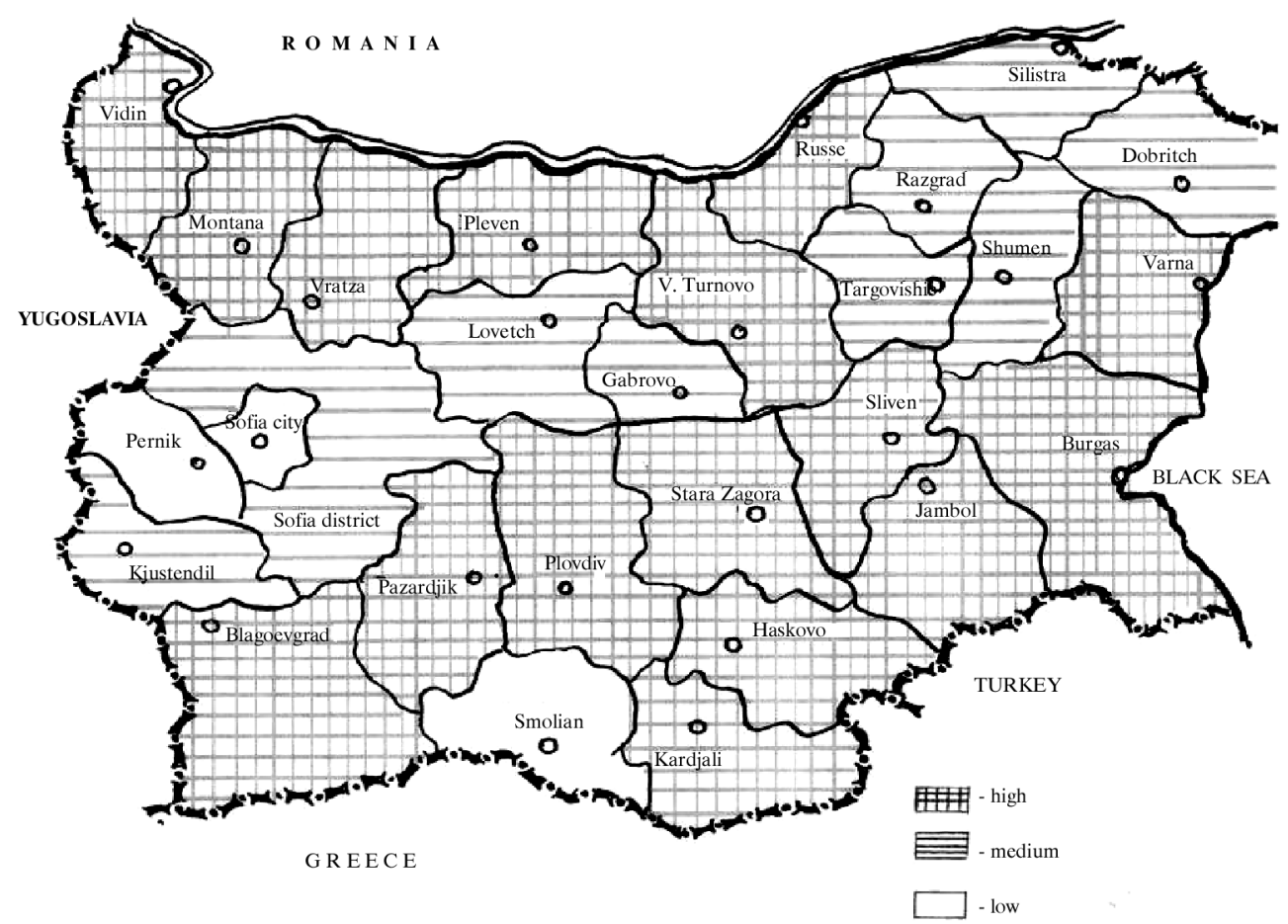

Figure 3. Risk of vivax malaria spreading in various regions of Bulgaria 
till 1999. It was supervised and controlled by the Ministry of Health, the whole public health network and particularly the primary health care taking part in the fulfillment of the planned measures. The activities provided for in the system are principally based on: chemoprophylaxis of Bulgarian citizens leaving for endemic countries; instruction of travelers about prevention of malaria infection conducted in the Hygiene epidemiological inspectorates; registration of persons arriving in Bulgaria from countries with endemic malaria and notification of the primary health care as to the place of dwelling and job; examination for malaria on basis of clinical and epidemiological indications of the registered groups; follow up of all travelers coming from countries with malaria (coding in the personal medical record) by primary health care units; obligatory hospitalization and radical treatment of all acute phase patients and parasite carriers; epidemiological and entomological investigation of each reported case of malaria and implementation of appropriate measures regarding the source and the vector in the focus; follow up study of the density and susceptibility of Anopheline mosquitoes to the insecticides administered; prompt notification of the Ministry of Health for each reported or suspected case of malaria.

Since 1999, there is no compulsory registration and follow up of all Bulgarian citizens arriving from countries endemic for malaria, as the border medical installations which had provided information before were closed. The measures mentioned above are carried out only regarding persons who seek pretravel medical advice and posttravel assistance. This limitation of activities regarding malaria surveillance on the background of the data presented above may create an additional risk of reintroduction of malaria in the country in cases of delayed diagnosis and treatment of persons with imported malaria.

The analysis of the essential malaria import in Bulgaria, climatic conditions and presence of Anopheline species revealed a fairly high level of malariogenic potential. There is a trend of malaria import rise, as Bulgaria is the first barrier for the large amount of immigrants coming from malaria endemic areas of the Near East, Africa and Asia who passed the country in their way to Europe. The increased population migration from and to Turkey should also be taken into con- sideration. All this determines the potential spreading of malaria infection over considerable parts of the country, differing in endemicity depending on climate, fauna and socio-economic factors. The renewed malaria transmission that resulted in an outbreak in a former endemic region in Southern Bulgaria convincingly shows a tangible possibility of reintroduction of malaria into Bulgaria and is as an indication for existence of potential risk of spread of indigenous malaria, especially in cases of delayed diagnosis and treatment. The new trends in the malaria situation, the global warming of the planet that may cause enlargement of the potential malaria season and risk areas in Bulgaria, the intensified international relations after the political changes and democratization of the country and the increased population migrations, require intensified efforts of the whole health network regarding control measures and surveillance of malaria in Bulgaria.

The last decades a general qualitative deterioration of the malaria situation in the world was recorded. There is a spread and intensification of parasite resistance to antimalaria drugs, new epidemics are reported - some of them in countries that have, until recently, been free of the disease (Najera et al., 1998; Najera, 1999; WHO, 1998, 1999; 2000; Ejov, 2001). In the European region, Azerbaijan, Tajikistan and Turkey have suffered explosive and extensive epidemics. Outbreaks have been registered in Armenia, Turkmenistan and Georgia (Ejov, 2001).

The highly endemic Balkan countries in the past are now facing a potential risk of reintroduction of malaria (Ejov, 2001; Gratz, 2001; Andreas, 2001; Nicilescu et al., 2001, Velo, 2001; Samanidou-Voyadjioglow, 2001; Karanis, 2001; Zgomba, 2001; Sapounas, 2001; Vakalis, 2001).

The measures carried out during the malaria outbreak eradication in 1995-1996 showed a lack of epidemiological, diagnostic and operational readiness for fast antimalaria activities of the health network. This requires the elaboration of surveillance and control system including prevention measures and activities in case of new indigenous transmission. Malaria is an endemic disease and the application of delayed or inadequate measures regarding the primary autochthonous cases will lead to serious health and socioeconomic problems. 
Prevention of malaria requires efforts of the Ministry of Health, National Center of Infectious and Parasitic Diseases, Public Health and Primary Health Care networks directed at efficient health education concerning health risk associated with travel and the need to obtain pretravel medical advice including chemoprophylaxis. Health-care providers and other professionals should be educated regarding optimal and accurate malaria prevention recommendations and to be aware of the necessity of prompt diagnostic evaluation in cases where symptoms of malaria occur after travel.

\section{CONCLUSIONS}

The analysis of malaria situation revealed new trends in the epidemiological features of the disease on the background of the socio-economical, climatic and ecological changes in the past years. The data showed a presence of a fairly high level of malariogenic potential and risk of reemergence of the disease in the country. The deteriorated malaria surveillance measures may also bring about the reintroduction of the infection.

The present situation, that probably will be valid for the future, too, requires a scientific based prognosis and improvement of a system of surveillance activities aiming at prevention of indigenous endemic and epidemic spreading of malaria in the country, as well as elaboration of adequate activities of the health network in case of reappearance of autochthonous cases.

\section{REFERENCES}

Andreas, H. (2001), Present malaria status and mosquito control methodologies in Cyprus, In: Proceedings of the

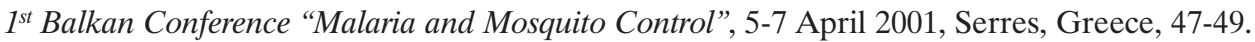

Christova, T. (1980), Epidemiological importance and geographical distribution of Anopheline mosquitoes in Bulgaria, Vector and rodents control Bulletin, 143, 3-6 (in Bulgarian).

Ejov, M. (2001), Malaria situation in European region, In: Proceedings of the $1^{\text {st }}$ Balkan Conference "Malaria and Mosquito Control”, 5-7 April 2001, Serres, Greece, 20-26.

Gratz, N. (2001), The risk of resurgent malaria in the Balkans, In: Proceedings of the $1^{\text {st }}$ Balkan Conference "Malaria and Mosquito Control", 5-7 April 2001, Serres, Greece, 27-32.

Karanis, P. (2001), The history of malaria in Europe and Balkan countries, In: Proceedings of the $1^{\text {st }}$ Balkan Conference "Malaria and Mosquito Control", 5-7 April 2001, Serres, Greece, 18-19.

Kurdova, R., Petrov, P., Jordanova, D., Vutchev, D. and Dinev, D. (2000). Status and control of parasitic diseases in the country and activities of the specialized parasitological network in 1999, NCIPD Information Journal, 5, 4-33 (in Bulgarian).

Najera, J.(1999), A. Malaria control. Achievements, Problems \& Strategies, WHO, Geneva, 1999,126 pp.

Najera, J.A., Kouznetsov, R. L, and Delacollette, C. (1998), Malaria epidemics. Detection and control. Forecasting and prevention, WHO, Geneva, $81 \mathrm{pp}$.

Nicolescu, G. et al. (2001), Evaluation of the risk of malaria reintroduction in Romania, In: Proceedings of the $1^{\text {st }}$ Balkan Conference "Malaria and Mosquito Control", 5-7 April 2001, Serres, Greece, 43-46.

Petrov, P. (1979), Experimental, diagnostic and epidemiological investigations of some indigenous and tropical parasitic diseases. D.Sc. Thesis, 422 pp. (in Bulgarian).

Petrov, P. (1990), Malaria. Medizina i Fizkultura, 128 pp. (in Bulgarian).

Petrov, P. (1997), Malaria-past and present problems. NCIPD Information Journal, 1, 4-10 (in Bulgarian).

Petrov, P. and Kurdova-Mintcheva, R. (1984), Malaria situation in the People's Republic of Bulgaria and control measures (1980-1983), WHO Malaria Co-ordination Meeting in South-East Europe, Athens, Greece.

Petrov, P. and Kurdova-Mintcheva, R. (1987), Epidemiological situation of malaria in Bulgaria (1984-1986), WHO Malaria Co-ordination Meeting in South-East Europe, Ismir, Turkey.

Petrov, P., Kurdova, R., Vutchev, D. and Jordanova, D. (2000), Epidemiological and medico-geographical characteristics of the imported in Bulgaria parasitic diseases in the period 1990-1999, In: Proceedings of the $4^{\text {th }}$ Congress of Medical Geography, Sofia, 94-96 (in Bulgarian).

Petrov, P., Kurdova, R., Vutchev, D., Jordanova, D. and Kalaidjieva, A. (2000), Analysis of parasitic diseases and of parasitological units' activities in the country in 1998, NCIPD Information Journal, 2, 5-15 (in Bulgarian).

Samanidou-Voyadjoglow A. (2001), Malaria vectors in Greece, In: Proceedings of the $1^{\text {st }}$ Balkan Conference "Malaria and Mosquito Control”, 5-7 April 2001, Serres, Greece, 62-65. 
Sapounas T. (2001), The current state of malaria in Greece-the policy of prevention, In: Proceedings of the $1^{s t}$ Balkan Conference "Malaria and Mosquito Control", 5-7 April 2001, Serres, Greece, 46.

Stanchev P. and Todorov, T. (1967), Malaria in Bulgaria. Medizina i Fizkultura, Sofia, 100 pp. (in Bulgarian).

Vakalis, N. (2001), Current methods of diagnosis and prophylaxis, In: Proceedings of the $1^{\text {st }}$ Balkan Conference "Malaria and Mosquito Control", 5-7 April 2001, Serres, Greece, 16-17.

Velo, E. (2001), Malaria and the sensitivity of anopheles to insecticides in Albania, In: Proceedings of the $1^{\text {st }}$ Balkan Conference "Malaria and Mosquito Control", 5-7 April 2001, Serres, Greece, 33-34.

Vutchev, D. and Kurdova, R. (1999), Clinical and parasitological aspects of Malaria tropica following splenectomy, Problems of Infectious and Parasitic Diseases, 1, 17-18.

WHO, (2001), International Travel and Health; Vaccination Requirements and Health Advice, Geneva, 99 pp.

WHO, Roll Back Malaria Partnership Meeting for Central Asian Republics and Kazakhstan, Report on a WHO Meeting. Tashkent, Uzbekistan, 8 June, 1999, 26 pp.

WHO, Roll Back Malaria, RBM A global partnership. WHO, Geneva, 1 September, 1998, 52 pp.

WHO, Roll Back Malaria, RBM Action at country level, Country Updates, October 1998-June 2000. WHO /CDS/RBM/200.24, $80 \mathrm{pp}$.

Zgomba M. (2001), Potential vectors of malaria in Yugoslavia, In: Proceedings of the $1^{\text {st }}$ Balkan Conference "Malaria and Mosquito Control", 5-7 April 2001, Serres, Greece, 61. 